\title{
EXPERIENCIAS Y VÍNCULOS CON EL SABER UNIVERSITARIO POR PARTE DE ESTUDIANTES EGRESADAS DEL PLAN FINES
}

\author{
Santiago Garriga Olmo* \\ CONICET - Universidad Nacional de La Plata, Argentina \\ santiago.garriga@hotmail.com
}

Recibido: 3/08/2020 - Aceptado: 28/12/2020

\section{Resumen}

El trabajo analiza las experiencias y los vínculos con el saber en el primer año de las carreras de Sociología y Enfermería de la Universidad Nacional de La Plata por parte de estudiantes egresadas del Plan FinEs. Se trata de una investigación de carácter cualitativo en la que se priorizó la técnica de la entrevista en profundidad. Describimos las características del Plan FinEs -y las experiencias de las estudiantes en dicha instancia- como así también algunas de las características de las carreras de Sociología y Enfermería con el fin de comprender el contexto en el que se desarrollan las experiencias educativas. El trabajo presenta el análisis de la información construida sobre la base del trabajo de campo de las experiencias y vínculos con el saber universitario a partir del primer acercamiento a la carrera, el curso de ingreso, el rol y las prácticas docentes y los textos y contenidos abordados en las materias de primer año.

Palabras clave: Experiencias - Saber - Ingreso - Plan FinEs - Universidad.

\section{EXPERIENCES AND LINKS WITH THE UNIVERSITY KNOWLEDGE BY GRADUATE STUDENTS OF THE "FINES PLAN"}

\begin{abstract}
The following work analyzes the experiences and links to knowledge by graduate students of the "FinEs Plan" in their first-year approach to Sociology and Nursing careers at La Plata National University (UNLP). This work tended to focus in qualitative investigation by prioritizing the in-depth interview technique. Furthermore, main aspects about "Plan FinEs" will be described as well as some characteristics of Sociology and Nursing careers, in order to understand in a deeper way, the context in which educational experiences are developed. Finally, it will be shown the analysis about information built based on fieldwork experiences and links with university knowledge from first approach to the

\footnotetext{
*Profesor y Licenciado en Sociología (UNLP). Doctorando en Ciencias de la Educación (FaHCE-UNLP). Becario Doctoral de CONICET con sede en el Instituto de Investigaciones en Humanidades y Ciencias Sociales (IdIHCS). Ayudante Diplomado en la asignatura Introducción al Proceso Intelectual de la Facultad de Ciencias Médicas (UNLP). Profesor en el Curso Introductorio de las carreras de Sociología (FaHCE-UNLP). Profesor en el Curso Introductorio en la Facultad de Ciencias Médicas (UNLP). Ayudante en el Seminario Análisis de Políticas y Sistemas Educativos.
} 
career, the entrance course, teaching practices, roles, texts and contents addressed in first-year subjects

Keywords: Experiences - Knowledge - Entrance course - "Plan FinEs" - University/Collage.

\section{Introducción}

El artículo analiza las experiencias y los vínculos con el saber por parte de estudiantes de las carreras de Sociología y Enfermería de la Universidad Nacional de La Plata que terminaron los estudios secundarios a través del Plan FinEs (en adelante FinEs). El propósito es conocer y describir las experiencias y el vínculo con el saber por parte de estudiantes que terminaron los estudios secundarios a través de un plan educativo que "se limita a la terminalidad educativa" (De la Fare, Rovelli y Atairo, 2016, p.17) y que posee características disímiles a las de la escuela tradicional.

Se trata de una investigación de carácter cualitativo en la que se priorizó la técnica de entrevistas en profundidad ya que nos permiten "acceder a la perspectiva de los actores, para conocer cómo ellos interpretan sus experiencias en sus propios términos" (Marradi, Archenti, Piovani, 2012, p.196).

Elaboramos un recorte tempo-espacial para enfocar el análisis en el inicio de las carreras: el curso de ingreso y el primer año. La literatura sobre el tema coincide en señalar que el primer año es un período de incertidumbre y el momento más crítico (entre otros Ezcurra, 2011; Pierella, 2014) y, según Más Rocha, "una instancia que posee un carácter esencialmente formativo, dado que la manera en la cual los estudiantes transitan su primer año en la universidad resulta fundamental para definir su futuro académico" (2019, p. 83). Para Bracchi, ingresar a la universidad

implica un cambio que no es solamente en términos institucionales o curriculares sino se ponen en juego los esquemas de percepción, de apreciación, de valoración y de acción que harán posible diversas tomas de decisiones contribuyendo a conformar el oficio de ser estudiante. Dicho oficio se conforma apropiándose de las reglas del juego propias de la institución y para ello deberá utilizar todo su capital económico, cultural y simbólico (Bracchi, 2016, p. 5).

Más allá de las particularidades de cada caso hay otra característica que atraviesa a cada estudiante: la acumulación de desventajas al momento de iniciar la carrera (Saraví, 2015). En este sentido, el interés por abordar las experiencias con el saber se articula con otra preocupación que es la de la permanencia de las estudiantes en la universidad ya que partimos de considerar que "la enseñanza es un condicionante primario, en cualquier tipo de alumnos, pero sobre todo en población de estatus de desventaja" (Ezcurra, 2011, p.53).

Durante el trabajo de campo realizamos seis entrevistas en profundidad a estudiantes de las carreras de Sociología y de Enfermería, todas mujeres cuyas edades oscilan entre los 23 y 53 años que finalizaron sus estudios secundarios entre 2015 y 2017 e ingresaron a la universidad entre 2017 y 2018. Las experiencias transcurrieron en dos facultades y disciplinas de conocimiento distintas y, también con un perfil de estudiante disímil.

El territorio teórico de la investigación se nutre de los aportes de la sociología educativa y en particular de aquella que hace foco en las desigualdades (Ezcurra, 2011; Saraví, 2015), de los estudios sobre la experiencia educativa (Carli, 2012; Bracchi, 2016; Pierella, 2011, 2014) y de los trabajos sobre la educación superior centrados en la problemática de los primeros años (Ezcurra, 2007; Pierella, 2016, Cerezo, 2017; Más Rocha, 2019).

Entendemos a las experiencias como "aquellos acontecimientos que les suceden a los sujetos, que los conmueven, los alteran, les dejan huellas, los transforman" (Brito et. al. Citado en Cerezo, 2017, p.75). Esta categoría nos permite "poner en suspenso las enunciaciones universalistas tan características del discurso universitario" (Carli, 2012, p.25). Consideramos que analizar las experiencias en los vínculos con el saber nos permitirá entender "las prácticas de los sujetos institucionales y sus reflexiones retrospectivas sobre lo vivido para poner en cuestión una mirada generalista, introducir cierto realismo en los modos de pensar la universidad y narrar la historia del presente" (Carli, 2012, p.26). Teniendo en cuenta que "la exploración de las experiencias de conocimiento de los estudiantes (...) revela la politicidad de las prácticas, su notable complejidad tanto desde el punto de vista material como subjetivo" (2012, p.136). Mientras que nos referimos a los saberes universitarios como aquellos saberes que no sólo se intentan transmitir desde las facultades y los/as docentes, sino también a "las relaciones interpersonales que tienen lugar en el enseñar y aprender, a las prácticas que se desarrollan para la adquisición de esos saberes" (Cerezo, 2017, p.78).

En primer lugar, describimos las características del FinEs -a fin de entender sus peculiaridadesy de las trayectorias socioeducativas de las estudiantes. Luego, hacemos una descripción de las carreras de Sociología y Enfermería con el fin de comprender el contexto en el que se desarrollan las experiencias educativas. A continuación, desarrollamos el análisis del trabajo de campo sobre las experiencias y vínculos con el saber universitario a partir del primer acercamiento con los contenidos y textos de la carrera, la experiencia en el curso de ingreso, el rol y las prácticas de los/as docentes y los textos y contenidos trabajados a lo largo del primer año. Por último, presentamos las reflexiones finales. 


\section{Características del Plan FinEs y las estudiantes}

En 2008 se lanzó el Plan de Finalización de los Estudios Secundarios destinado a personas mayores de 18 años que no hubieran terminado sus estudios secundarios. El objetivo fue llevar adelante "la implementación de un Plan específico, adaptado a sus posibilidades y necesidades" (Ministerio de Educación de la Nación, 2011, p.8).

Cuando se implementó contaba con un régimen de cursada presencial y una carga horaria de ocho horas semanales durante dos días, la asistencia obligatoria de los/as estudiantes al $75 \%$ de las clases y con dos orientaciones en el plan de estudios: Ciencias Sociales y Gestión y Administración (Cuestas, 2014).

El FinEs también se caracteriza por la gran cantidad de actores que intervienen en su ejecución: desde el Estado Nacional que aporta los fondos hasta organizaciones sociales que, con autorización estatal, tienen facultades de abrir sedes. Esto permite que muchas veces se abren sedes del FinEs en barrios donde no hay escuelas para adultos (Finnegan y Brunetto, 2015). A su vez, los lugares donde se dictan las clases distan mucho de ser espacios diseñados y construidos para estudiar ya que pueden ser casas familiares, delegaciones municipales, clubes de barrio, etc.

Algunos trabajos reflejan las controversias y puntos contradictorios al momento de analizar el FinEs y sus actores y destacan "la falta de trabajos que analicen la articulación de los distintos niveles de enseñanza y las trayectorias postescolares de los/as egresados/as" (Garriga Olmo y Torres, 2018, p. 43).

Las estudiantes consideran que, dadas sus condiciones, el FinEs fue la única manera de terminar los estudios secundarios. Destacan la flexibilidad en el régimen de cursada -que les permitió combinar estudios con actividades laborales y familiares-, la cercanía del lugar de estudio y el "ambiente" que se generaba, tanto entre los/as estudiantes -casi siempre en las mismas condiciones-como con los/as docentes.

Las estudiantes aquí entrevistadas no cuentan con familiares directos que hayan transitado por la Universidad y la posibilidad de acceder y estudiar una carrera se les presentó "de grandes". En todos los casos se trata de estudiantes no tradicionales para el mundo universitario (Linne, 2018) y cuyas trayectorias educativas se vieron interrumpidas en algún momento: ingresaron a la Universidad sin la progresión lineal prevista por el sistema educativo (Terigi, 2008). Por ser la primera generación universitaria "se trata de novatos, inexpertos, que con frecuencia tienen poca idea de qué esperar y una escasa comprensión sobre cómo el ambiente académico puede afectar sus vidas, y que por añadidura deben enfrentar una autodirección considerable" (Ezcurra, 2007, p.14). También hay que señalar que acumulan desventajas si consideramos sus situaciones laborales, composición familiar y "diversos accidentes biográficos" (Saraví, 2015, p.32) como son el hecho de haber interrumpido en algún momento sus trayectorias educativas, ser madres, contar con trabajos precarios y vivir en la periferia de la ciudad.

Las estudiantes, en términos generales, valoran mucho la experiencia en el FinEs. Sin embargo, al momento de empezar a cursar en sus respectivas carreras, señalaron dificultades para afrontar las lecturas y algunos temas, explican que en la universidad tuvieron que enfrentarse a un nuevo ritmo de estudio, para el cual no se sentían preparadas, y estudiar temas "nunca vistos". Ante la experiencia universitaria la valoración de los saberes se resignificó: lo que aprendieron en el FinEs pudo ser importante y útil en algunos aspectos pero fue "básico" y "poco exigente" de cara a estudiar una carrera universitaria.

Bourdieu (1997) utiliza el concepto de capital cultural como aquellas disposiciones y hábitos adquiridos por los individuos en el proceso de socialización. Aunque coincidimos en que cualquier ingresante novato en la Universidad llega sin experiencia en el nivel, el capital cultural -que remite a los saberes- facilita o dificulta dicha experiencia. Dichos saberes tienen dos aristas: "saber pensar (pensar, entender vs. repetir, memorizar); y saber aprender, saber estudiar; por ejemplo, tomar apuntes en clase, cómo estudiar mucha bibliografía y comprenderla, cómo organizar el tiempo y las actividades, cómo preparar los exámenes" (Ezcurra, 2007, p.33). Esos saberes son muchos y variados: saber usar la biblioteca, saber organizar el tiempo de estudio, saber hacer un resumen y tomar apuntes, entre otros. Como veremos a continuación, los cursos de ingreso diseñados en cada carrera son un intento por enseñar y que los/as estudiantes incorporen dichos saberes ya que la Universidad se presenta como un espacio nuevo y diferente no sólo en términos sociales y geográficos, sino fundamentalmente en lo que hace a las experiencias y vínculos con el saber.

\section{Sociología y Enfermería: características de las carreras y del curso de ingreso}

Las experiencias analizadas transcurren en dos unidades académicas y de disciplinas diferentes, con características sociodemográficas y organizativas disímiles pero que comparten similitudes sobre las políticas, principalmente el curso de ingreso, destinadas a aquellos/as estudiantes que ingresan por primera vez a la educación superior.

La carrera de Sociología -tanto el profesorado como la licenciatura1 - fue inaugurada en 1993. Sin ser una carrera tradicional dentro de la UNLP tampoco es nueva. Tiene un plan de estudios de cinco años con seis materias cuatrimestrales en el primer año, se dicta en la Facultad de Humanidades y Ciencias de la Educación (FaHCE) y es una carrera "chica": en 2017 tuvo 230 ingresantes. Sus estudiantes se destacan por ser jóvenes y provenientes de familias con un clima educativo alto (Cotignola et. al., 2017).

\footnotetext{
${ }^{1}$ A los fines de este trabajo nos referimos indistintamente al profesorado y la licenciatura.
} 
Por su parte, la carrera de Enfermería Universitaria pertenece a la Facultad de Ciencias Médicas (FCM) y se inauguró en 2016. Cuenta con un plan de estudios de tres años con siete materias en el primer año -seis cuatrimestrales y una anual-. En 2018 ingresaron 674 estudiantes de las cuales el 85\% fueron mujeres y el $50 \%$ son madres (El Día, 11 de marzo de 2018).

Como se puede apreciar, el perfil académico de cada carrera y las características de sus estudiantes son diferentes. Sin embargo, ambas contemplan un curso obligatorio para quienes ingresan por primera vez a la universidad que tiene el propósito de atender a la articulación entre el nivel medio y superior y así facilitar el acceso y la ambientación al mundo universitario. Las ingresantes a Enfermería, al momento de inscribirse recibieron un cuadernillo con ejercicios de biología, físico-química y matemática para entregar completado una vez comenzado el curso. No así las ingresantes de Sociología que también trabajaron con un cuadernillo pero una vez iniciado el curso.

El curso de Sociología se denomina Curso Introductorio, tiene un mes de duración de diez horas semanales, no es eliminatorio y tiene el objetivo de articular y facilitar el tránsito del nivel secundario al nivel universitario. En Enfermería el curso se denomina Curso Introductorio a la Vida Universitaria y los/as estudiantes asistieron dos veces por semana durante un mes con clases de dos horas. Los dos cursos abordan la comprensión y producción de textos y presentan su preocupación por introducir a los/as estudiantes en la vida universitaria. El cuadernillo de Enfermería hace hincapié en dicho aspecto y señala:

entendemos que, para garantizar el derecho a la educación pública, gratuita y de calidad, es necesario pensar y ofrecer dispositivos que permitan a los y las ingresantes herramientas iniciales que promuevan no sólo el ingreso, sino la permanencia y el egreso (...), generando una trayectoria educativa que efectivice los conocimientos que desde dicho establecimiento nos proponemos brindar (Escuela Universitaria de Recursos Humanos del Equipo de Salud, 2017, p. 1).

Por lo tanto, más allá de las diferencias entre una carrera y otra, encontramos que ambas comparten la preocupación y el interés por la ambientación de los/as estudiantes a la vida universitaria y la decisión de establecer un curso de ingreso para dicho objetivo.

\section{Trayectorias educativas y formación previa}

Un aspecto sustancial recogido del trabajo de campo tiene que ver con la interrupción de las trayectorias educativas y cómo afecta en las experiencias universitarias. Aunque los motivos de interrupción no sean idénticos, sí podemos asegurar que las estudiantes lo vivieron como un obstáculo. También señalaron que terminar sus estudios en el FinEs fue "insuficiente" para encarar la carrera y esta valoración acerca de los saberes aprendidos surgió a partir de la experiencia universitaria, no antes.

El cuadernillo con ejercicios de matemática, biología y físico-química fue el primer contacto con el saber universitario para las estudiantes de Enfermería. Para ninguna fue fácil completar los ejercicios y las razones las encuentran en la formación previa.

- Yo creo que el FinEs no te prepara para la Facultad. ¿Sabés cuándo me di cuenta? Cuando tuve que hacer los cuadernillos que nos dieron para el comienzo (Eva, estudiante de Enfermería).

La experiencia que relata Carina $^{2}$ en la carrera de Sociología también estuvo atravesada por el sentimiento de no estar a la altura de lo que demanda la Universidad:

- Sentía que no estaba preparada (...) Cuando arrancó el curso me presenté con todo, cuando presentaron el cuadernillo y demás cosas dije 'chau, no entiendo nada.

A su vez, observamos la incidencia que adquiere en las experiencias universitarias y los vínculos con los saberes el hecho de haber interrumpido sus trayectorias educativas.

Después química hemos hecho muy poco (...) Lo hizo mi hermana porque ella entendía.

¿Tu hermana por qué entendía de química?

Y porque ella fue al FinEs pero salió de la escuela y se metió en el FinEs. Yo salí de la escuela y [mucho tiempo] después me metí al FinEs.

¿Creés que se nota la diferencia?

- Un montón. Lo que yo no entendía de matemática ella se acordaba más. Y yo decía no puede ser. Y claro si ella había terminado el colegio y como tuvo un hijo lo dejó, ni lo dejó tampoco porque quedó embarazada y lo hizo embarazada también el FinEs. Yo dejé de estudiar y retomé al año, a los dos años. Llega un momento en que si uno no estudia después no se acuerda. (Rocío, estudiante de Enfermería).

\footnotetext{
${ }^{2}$ Todos los nombres fueron modificados con el propósito de mantener el anonimato de las estudiantes entrevistadas.
} 
En una situación similar a la suya -con un hijo a cargo y habiendo terminado la secundaria en el FinEs-, Rocío resalta que su hermana no tuvo inconvenientes para completar el cuadernillo porque "entendía".

- Me gustó menos lo que es Química porque hace años que no agarro una cuenta.

¿Tuviste química en el FinEs o algo parecido?

No, matemática nada más.

O sea, lo que estás viendo de química en la Facultad no lo veías ¿desde hace cuánto tiempo?

- Y añares... más de ocho años.

Por último, las estudiantes reconocen algunas desventajas con respecto a sus pares que terminaron la secundaria en escuelas tradicionales.

Yo estaba en un grupo [de Whatsapp] de Enfermería oficial [organizado por el Centro de Estudiantes] (...) Te iban explicando la realización del cuadernillo... los chicos que habían terminado el secundario, por lo que ponían ahí, era re fácil. Había gente que había terminado el colegio hacía 10 años, 15 años y no entendían nada y yo que había terminado este año pero tampoco, estaba como si lo hubiese terminado hace 10 años, porque no tuve química, [de] matemática lo que tuve fue muy básico, ecuaciones, fracciones pero básico. Y en el cuadernillo era bastante más elevado. Tuve que contratar un profesor particular de matemática para que me explique (Eva, estudiante de Enfermería).

Este testimonio refleja que, ante la desventaja inicial de no saber ni poder resolver los ejercicios, se añadió una desventaja nueva: resolver las falencias académicas de manera individual y por fuera del ámbito universitario.

Las experiencias reflejan las dificultades que se presentaron para las estudiantes que terminaron la secundaria en el FinEs y los problemas que detectaron dada la interrupción de sus trayectorias educativas. En este sentido, la imposibilidad de transitar los estudios secundarios pero sin avanzar por las trayectorias teóricas del sistema educativo (Terigi, 2008) dificulta los aprendizajes de las estudiantes.

\section{El primer acercamiento a la Universidad}

Otro aspecto importante en la experiencia de las estudiantes tiene que ver con su carácter de inexpertas del mundo universitario. Esto se manifiesta en vergüenza y "admiración" por los profesores y compañeros/as que las inhibe a preguntar sobre algo que no entienden en clase. En el relato de Alicia se refleja de la siguiente manera:

- $\quad$ Cuando no entendías algo en clase, ¿preguntabas para sacarte las dudas?

- No, me daba vergüenza. Imagínate las cosas que opinaban los pibes. Le digo a mi hija, a mí ni se me ocurriría pensar eso. Tienen un bocho los pibes.

(Alicia, estudiante de Sociología).

Otra estudiante señaló que la fascinación por estar en la universidad la dejaba en una posición completamente pasiva:

Yo ahí en el curso de ingreso me sentía... mirá que yo hablo un montón y soy muy sociable, pero me sentía como que era espectadora mirando todo, y quería saber, más que nada informarme bien, no sea cosa que hable y meta la pata, ese era el miedo mío. Pero porque no me soltaba yo (Silvia, estudiante de Sociología).

Para Rocío el miedo a "decir cualquier cosa" también estuvo muy presente en las primeras clases:

Yo era callada, no hablaba con nadie, no tenía trato con nadie. No quería hablar con nadie por miedo a decir algo que esté fuera de lugar y quedar como una tarada (Rocío, estudiante de Enfermería).

Sin dudas que las expectativas y el aprendizaje de cómo actuar en la universidad adquieren un rol fundamental en las primeras clases. La experiencia de Rocío, que antes de estudiar Enfermería cursó un año de Trabajo Social, sirve de ejemplo:

- El primer día en Trabajo Social yo estaba perdida. Y en Enfermería yo ya tenía un poquito más de cancha.

- $\quad$ La experiencia en Trabajo Social te sirvió...

- Sí, bastante (...) Porque después cuando vine acá preguntaba cualquier cosa, no me importaba nada. 
Estas experiencias muestran que la ambientación a la vida universitaria son un factor clave para el desenvolvimiento y la acción de cada estudiante. La posibilidad de hablar en clase, preguntar algo que no se entiende, parece clausurada por el temor a decir algo "fuera de lugar" o habilitada dado el conocimiento y las experiencias previas.

\section{El curso de ingreso}

Respecto a la ambientación, el curso de ingreso juega un rol clave en la experiencia de las estudiantes: permite conocer compañeros/as de la carrera. Sin ser un aspecto indispensable, la experiencia de Eva en una de las primeras clases después del curso de ingreso da cuenta de la tranquilidad que brinda estudiar con personas medianamente conocidas.

Está bueno entrar y ver caras conocidas, como que entré más relajada (...) Entré media tensionada y vi que este grupito [compañeros/as del curso de ingreso] que estaba ahí y dije "ah bueno" (Eva, estudiante de Enfermería).

El imaginario de lo que implica estudiar en la Universidad también adquiere un rol central en este primer acercamiento. La falta de conocimientos sobre la modalidad de estudio en el nivel universitario puede llevar a situaciones frustrantes y desconcertantes. La importancia que implica tener parientes o amigos/as que hayan pasado por la Universidad se vuelven relevantes. Rocío cuenta cuál era su expectativa de una clase en la facultad y lo que finalmente se encontró:

¿Cómo imaginabas que era estudiar en la Facultad?

Como estudiar en la primaria. [Pero] Nada que ver, era más exigente. Como que no tenés vida social (...) Yo me metí de lleno en la Facultad pensando "bueno, acá te van a explicar, te van a enseñar" y no, ahí si no te hacés vos nadie va a ir y te va a explicar y se va a sentar a explicar.

"Hacerse uno" da cuenta de lo solitario que resultó ese aprendizaje. Observamos que, sin referentes a los que recurrir en sus entornos más cercanos y en un ámbito donde esos saberes quedan explicitados, la tarea de saber cómo manejarse en la Universidad recayó sobre cada una. Esto mismo ocurrió con lo que esperaban encontrarse:

¿Cómo te imaginabas que era la Facultad antes de empezar? ¿Te imaginabas algo parecido al FinEs, o muy distinto?

- $\quad$ No me imaginaba nada. No sé, no pensé en nada. Creo que quería vivir la experiencia y la viví.

Las condiciones económicas son un factor importante al momento de analizar experiencias y trayectorias educativas, pero no las únicas. Retomando a Saraví, existen otros factores sumamente relevantes para nuestro análisis: "Deficiencias de capital social y cultural en los hogares, condiciones precarias de las viviendas, falta de soporte, contención y estímulo, transiciones laborales y familiares muy tempranas, dificultades para sostener proyectos de largo plazo, entre otros" (Saraví, 2015, p.66).

Las expectativas, lo que esperan encontrarse en la facultad es parte de las ventajas o desventajas con las que cuentan unos y otros, según su procedencia. Como cuenta Rocío, ella aprendió y entendió cómo era estudiar en la facultad una vez que ingresó. Nadie le advirtió con qué se iba a encontrar y eso fue una desventaja y un obstáculo a superar desde el comienzo.

Encontramos que las expectativas y el imaginario de "estudiar en la Facultad" estaban lejos de parecerse a lo que luego vivieron. Queda expuesta entonces la dimensión cultural de la desigualdad social, entendiendo dicha dimensión como "formas de actuar y de hablar, percepciones sobre los otros y uno mismo, estigmas y estilos de vida, preferencias, expectativas y prácticas cotidianas, entre muchos otros" (Saraví, 2015, p.41).

En la experiencia de las egresadas del FinEs, el proceso de aprendizaje y vinculación se reconoce por dos aspectos: por un lado, el saber académico y por otro lado, su experiencia y situación requirió que aprendieran el habitus universitario, entendiendo por habitus aquellas disposiciones adquiridas por las y los actores en un campo determinado. Como señala Bourdieu (2007), dichas disposiciones adquiridas están relacionadas con el conocimiento que las estudiantes pudieran tener del ámbito en cuestión, en este caso la Universidad. Dichas reglas no escritas, son transmitidas en muchos casos por las familias de cada estudiante pero, para este grupo de estudiantes, el habitus universitario les resultaba completamente ajeno.

[El curso de ingreso] Me re sirvió (...) Para conocer lo que es la vida universitaria...

¿Qué fue lo que aprendiste?

Aprendí a hacer resúmenes, cómo me voy a tener que manejar con el estudio. Saber que cada vez que leo un texto lo voy a tener que subrayar para después el día de mañana no tener que leer todo de nuevo. Tener ideas principales, me re sirvió.

Eso para estudiar, pero también decís para la vida universitaria, ¿de eso qué aprendiste?

- Y más o menos cómo van a ser las clases, más charladas, eso es lo que me llevo yo. Un aprendizaje. 


\section{(Eva, estudiante de Enfermería Universitaria).}

Las experiencias analizadas dan cuenta de la importancia que adquirió el curso de ingreso porque otorga aprendizajes significativos para las estudiantes. Podemos afirmar que el curso logra familiarizarlas con el mundo universitario, es sumamente valorado de cara al futuro y, al mismo tiempo, se vuelve importante en términos académicos.

De acuerdo a los testimonios, conocer compañeros/as de cursada se vivenció, rápidamente, como una ventaja apreciada por las estudiantes y que habilitó estrategias para entender algún tema durante las cursadas. Rocío cuenta que entre compañeros y compañeras de comisión tienen un "grupo de estudio". Es un grupo de whatsapp donde intercambian preguntas o saberes y resulta importante porque ahí "siempre hay un nerd que entiende más". Así la socialización con compañeros/as permitió desplegar estrategias en sus vínculos con el saber.

\section{El rol y las prácticas de los/as docentes}

Hay un actor fundamental en los vínculos que los/as estudiantes establecen con el conocimiento del mundo universitario y es el de los/as docentes. Ezcurra señala que: "los profesores en el aula constituyen la variable institucional más relevante en el desempeño estudiantil, tan decisiva que su incidencia es mayor que cualquier otro factor institucional" (Ezcurra, 2007, p.41). Según Pierella:

No hay relato de estas trayectorias que podamos considerar exitosas en el que no se haya mencionado alguna figura profesoral que haya operado como sostén a la institución o en el que al menos no se haya expresado un deseo de haber contado con ellos (Pierella, 2014, p.185).

Trabajos más recientes dan cuenta de la preocupación que despierta en algunas facultades, y lo problemático que resulta, la falta de compromiso de los docentes en los cursos de ingreso y primer año de las carreras (Gorostiaga, Lastra y Muiños de Britos, 2017). A su vez, un relevamiento del Ministerio de Salud de la provincia de Buenos Aires señala que para el 93\% de los/as enfermeros/as "un buen docente es lo que más incide en su formación" (2017, p.41) y plantea "propiciar nuevas estrategias y recursos didácticopedagógicos para lugar a la mejora de las instancias prácticas en el proceso de enseñanza-aprendizaje de la Enfermería como campo disciplinar" (2017, p.41).

Entonces la relación y el vínculo que las estudiantes puedan tener con sus docentes es un aspecto central para sus experiencias, aprendizajes y trayectorias futuras. Este fenómeno, que entiende al buen docente como aquel que logra relacionarse con los estudiantes, es lo que Abramowski (2014) define como pedagogía relacional, es aquella en la que el docente debe:

Relacionarse con todos sus alumnos, estableciendo vínculos con ellos en tanto personas (...) para que una pedagogía relacional sea posible, es necesario que exista un contacto cercano, que se acorten las distancias y se genere un espacio íntimo entre docente y alumno (Abramowski, 2014, p.92).

Podemos identificar que, durante el curso de ingreso, las estudiantes reconocieron y vivieron una modalidad de trabajo y un vínculo pedagógico con sus docentes que no fue igual durante las materias de primer año. En el curso los profesores aparecen representados por las preocupaciones que puedan tener por sus estudiantes, más allá de los saberes académicos. En algunos casos, las estudiantes mantuvieron un contacto con sus docentes por fuera de la cursada con el propósito de alentarlos a continuar la carrera o conversar sobre sus dificultades.

La manera de trabajar de cada profesor es sumamente significativa y las estudiantes saben juzgarlo rápidamente:

Hay gente en la Facultad que no está capacitada para ser profesor (...) porque yo para leer filminas me quedo en mi casa. [La profesora] leía filminas y a mí no me sirve. A mí me sirve que me enseñes porque encima yo tengo que ir a pagar un profesor particular para que me enseñe. Un desastre (...) La flaca es re buena, no te puedo decir que no, pero como profesora no va. Nos daba un tema y se perdía y decía "uh, para que no me acuerdo qué es". Iba a la filmina y lo buscaba. Yo sé que el presupuesto por ahí no alcanza para poner otro profesor, pero pongan alguien como la gente. Ellos dicen "estamos en la Facultad", bueno lo mismo digo yo: estamos en la Facultad. ¿Por qué tengo que sacar de mi bolsillo para ir a pagar un particular? (Rocío, estudiante de Enfermería).

El testimonio contrasta dos experiencias con docentes. Por un lado, aquellos que "enseñan al ritmo de los estudiantes". La contracara son aquellos profesores que "hablan, hablan y si entendiste, entendiste, $y$ sino jodete". De alguna manera, este testimonio parece indicar que, en algunos casos, son los/as propias docentes quienes no conciben las clases acorde a las necesidades y características de las estudiantes. La misma estudiante remarca la diferencia que hay con otro profesor que es "muy didáctico", también lee filminas "pero explica". El inconveniente no está en el recurso sino en la manera en que se lo utiliza. 
Definitivamente, el trabajo de cada docente, la manera de dar clase y la preparación es sumamente apreciado por las estudiantes.

El cúmulo de desventajas con el que ingresan las estudiantes a la Universidad puede acentuarse por las condiciones que ofrece la propia facultad. Los casos de profesores/as que solo "leen filminas" es sumamente problemático pero las mismas estudiantes destacan la contracara: los profesores que explican bien "porque se preocupan de que cada estudiante entienda el tema y la materia".

Según Carina, "el profesor condiciona al momento de estudiar (...) al momento de rendir, todo". Es interesante señalar que los/as profesores del curso de ingreso parecen desarrollar una tarea extra educativa: las "alientan", promueven el estudio, la lectura e invitan despejar dudas.

¿Qué recuerdos tenés del curso de ingreso?

Tuvimos un profesor y dos ayudantes. El profesor lo hacía bastante interesante, nos apoyaba mucho, nos incitaba a que estudiemos, a que leamos, a que sigamos la carrera, que no abandonemos. Era bastante positivo y nos daba bastante apoyo. Siempre estaba dispuesto a que cualquier duda que tengamos, algo que nos cueste se lo digamos. Que no nos quedemos callados con las dudas adentro. Que él estaba para enseñar, para ayudar también y siempre estaba dispuesto a que nosotros podamos aprender.

Esta situación aparece con menos frecuencia una vez avanzada la carrera. En las cursadas de las primeras materias emergen distintas evaluaciones sobre la labor docente. Los "copados" son aquellos que, principalmente dan buenas clases, pero también orientan las lecturas y el estudio para rendir parciales y explican "dos o tres veces si es necesario". Son quienes logran atraer la atención del alumnado y hacen más interesantes las clases aunque el tema o la materia puedan ser difíciles. Explicar bien los temas de la materia es el aspecto más destacado, más allá de los contenidos abordados:

Los teóricos de Filosofía eran un lujo, la profesora explicaba todo bastante bien con paciencia (Carina, estudiante de Sociología).

En contraposición a este tipo de docentes figuran aquellos que "en clase hablan de cualquier cosa", tienen una modalidad de trabajo en la cual "el alumno se tiene que acoplar a la clase que da el profesor".

Tenés profesores que te explican las cosas, por ejemplo, a veces te explican algo con un vocabulario bastante llano, por así decirlo. Y después tenés otro profesor que te explica el texto con el mismo vocabulario que está en el texto entonces no sabés qué carajo te está diciendo (Carina, estudiante de Sociología).

El distanciamiento entre profesor y estudiantes también es señalado como un aspecto negativo como así también las clases multitudinarias que aparecen como un obstáculo, no solo para la ambientación a la vida universitaria, sino también para el aprendizaje. Para Rocío "podés estudiar con 30, pero ya 100 o 200 es muy difícil". Otra problemática que emerge es la falta de precisión sobre los criterios docentes al momento de evaluar y tomar exámenes, algo que redunda en una desconfianza absoluta en las estudiantes al momento de presentarse a rendir.

La cantidad de estudiantes en el aula, la manera de dar clases del docente y el vínculo que los/as estudiantes logran establecer con éste aparecen como factores destacados. Las clases multitudinarias se presentan como un obstáculo mientras que las clases con menor cantidad de alumnas/os rememoran experiencias pasadas. Las primeras clases de Eva, luego del curso de ingreso, fueron con una cantidad de estudiantes similar a cuando cursó en el FinEs y para ella "está bueno".

\section{Textos académicos y nuevos aprendizajes}

Al indagar en el vínculo con los saberes del mundo universitario, una de las dificultades más recurrentes es el de los textos y escritura académica. Así lo que señala el equipo docente del curso de ingreso de Sociología: "nos encontramos con que la escritura, el ensayo de escritos propios, aparecía como uno de los principales problemas de los ingresantes" (Aréchaga et. al., 2015, p.10).

Los textos académicos presentan características disímiles a los textos trabajados muchas veces en el nivel secundario (Carlino, 2005). Los testimonios reflejan este quiebre entre lo que se lee en la Facultad y lo que estaban acostumbradas a leer en otros ámbitos. Aunque no excluye el interés por el tema, Carina asegura que "la forma de hablar y desarrollar que tiene los textos es bastante complejo para mî". En el caso de Silvia, la lectura que demandaba la Facultad era sumamente diferente a la del FinEs y esta diferencia, como también señala Alicia, está vinculada al lenguaje académico. Sin precisar los textos trabajados, una vez iniciada la carrera aparece una marcada diferencia entre aquellos textos difíciles, que vendrían a ser los teóricos, y aquellos más llevaderos y fáciles de entender que vendrían a ser textos "más prácticos".

Eva señaló que los textos vistos en las primeras clases fueron "interesantes" pero enseguida aclara "me di cuenta que tengo problemas para la comprensión de textos". A su vez, y prácticamente como una constante, la iniciación en los textos académicos va acompañada y aumenta su complejidad de temas nuevos. Las estudiantes no sólo se enfrentaron a nuevos formatos de lectura sino que también abordaron 
temas completamente novedosos en sus trayectorias educativas. Los textos se presentaron como "re pesados", sus contenidos "nuevísimos" y nunca estudiados anteriormente. Respecto al vocabulario de los textos cuenta Rocío que "he pasado noches buscando palabras en el diccionario".

Entendemos que estas experiencias habilitan un debate: ¿qué rol cumple la Universidad, los profesores y las diferentes cátedras en el aprendizaje de la lectura y escritura académica? Este debate excede los marcos de este trabajo, pero no deja de ser necesario abordar el tema porque, como observa Bracchi, "no se puede pretender que, sin las acciones pedagógicas necesarias, los estudiantes lean y escriban todo lo que les es ofrecido por las cátedras y que lo hagan en tiempo, en forma y correctamente" (Bracchi, 2016, p.12). La autora explica que adquirir habilidades sobre el modo de leer y escribir en la Universidad resulta fundamental para los/as estudiantes en general, pero sobre todo para quienes son primera generación universitaria. En definitiva, es la propia institución la que debe brindar a los estudiantes "las herramientas necesarias para acceder, permanecer y egresar" (Bracchi, 2016, p.13).

Cursar una carrera modificó hábitos y prácticas de estudio y también habilitó otras nuevas. Rocío explica que "antes hablaba así nomás" pero desde que comenzó a estudiar en la Universidad cambió su léxico "por la lectura, los profesores y las materias". También señaló su admiración por la manera de hablar de algunos docentes, la describe como "única", y por eso "hay cosas que dice y por ahí no las sé, entonces las tengo que buscar en el diccionario". Al mismo tiempo, el ingreso a la carrera no sólo modificó hábitos vinculados al estudio, sino también otro tipo de reflexiones y consideraciones de la vida cotidiana, como en el caso de las estudiantes de Enfermería.

Me cambió mucho la manera de pensar. (...) A ver cómo decirte... una enfermedad, si yo estaba enferma y decía "bueno, es normal que pase". Ahora es como que estoy más [atenta]... voy al hospital o si me pasa algo me hago atender. Antes por ahí no le daba mucha importancia.

Es importante entender que el vínculo con el saber de estas estudiantes excede los marcos y conocimientos que brinda la carrera. Utilizar el diccionario, preocuparse por la manera de hablar y modificar prácticas en la relación a la salud también fueron aprendizajes adquiridos en su experiencia universitaria y dichos aprendizajes absolutamente valorados por ellas mismas.

\section{Consideraciones finales}

En las experiencias relevadas se destacó la falta de articulación entre los contenidos de los temas -y materias- del FinEs y las carreras de Enfermería y Sociología. Las estudiantes experimentaron diversas dificultades en el ingreso a la universidad dado que cuentan con un bagaje de temas y contenidos que consideran "básicos". Otro factor que surgió como dificultad para entender los temas es la interrupción de sus propias trayectorias educativas. Esta combinación de factores se presentó como los dos elementos de obstáculo en el vínculo con el saber disciplinario de cada carrera.

En los casos de las estudiantes que ingresan por primera vez a la universidad, el carácter de inexpertas acerca del mundo universitario y el escaso capital cultural y social con el que ingresaron también influyó en los vínculos con el saber. Observamos que "el miedo de decir algo fuera de lugar" inhibió la participación o la posibilidad de realizar preguntas en clase a los/as docentes. Pero, como ya fue señalado en otros trabajos (Colabella y Vargas, 2014), aquí también encontramos un contraste destacado con el caso de aquellas estudiantes que cuentan con alguna experiencia universitaria previa.

En todos los casos, la internalización y el conocimiento de lo que implica la vida universitaria ocurrió en la Universidad misma y no antes. Podemos inferir que las estudiantes se enfrentaron a un aprendizaje doble: incorporar el oficio de ser estudiantes universitarias y aprender los contenidos de la carrera.

Para lo primero cumplieron un rol importante los cursos de ingresos de ambas carreras. Fue una instancia sumamente valorada para aprender el oficio de estudiantes y desplegar mayor capital social. Pero podemos señalar que, al mismo tiempo, el curso se presentó como una "isla" en sus trayectorias por tratarse de un espacio con características singulares que no se repitieron durante la cursada de las materias. Las estudiantes experimentaron una pedagogía relacional con sus docentes que no volvió a ocurrir una vez finalizado el curso.

Consideramos que los objetivos que las distintas unidades académicas se trazaron a partir del curso de ingreso pudo ser un mecanismo importante, pero sin la continuidad necesaria durante el primer año para estudiantes en desventaja. Pudimos reconstruir situaciones en las que las estudiantes debieron desplegar estrategias individuales para entender contenidos de la carrera.

En este sentido, aunque no fue el único aspecto mencionado, la práctica de los docentes sobresalió en los relatos. Nuestro trabajo refuerza los contenidos volcados en otras investigaciones que señalan la importancia de lo que ocurre adentro del aula, sobre el rol y prácticas desplegadas por el cuerpo docente de cada materia, principalmente en los primeros años (Ezcurra, 2007; Pierella, 2014). Observamos que, para las ingresantes, la manera de enseñar del docente fue sumamente importante dado que lo que ocurre en el aula es un momento decisivo para aprender los contenidos de la materia. Consideramos que la importancia radica en que, por las desventajas con las que ingresan a la universidad, los momentos para aprender no pueden ser desaprovechados.

Hay que tener en cuenta que las estudiantes manifestaron encontrarse en la universidad ante temas nunca antes vistos y textos que resultaron "re pesados" pero, más allá de las desventajas iniciales 
de las estudiantes, el rol del/a docente se presentó como un factor decisivo que ayudó u obstaculizó los aprendizajes. Así encontramos experiencias en las que las clases fueron "un lujo" siempre y cuando el/a docente explicara los contenidos al ritmo de los/as estudiantes y con buenas estrategias pedagógicas, como así también fuertes críticas porque las clases no ayudaron a entender los temas.

Otro aspecto a destacar es que no encontramos experiencias en donde las estudiantes abordan la lectura y escritura académica en las cursadas del primer año, algo sumamente relevante dadas las dificultades que manifestaron al respecto y que es, muchas veces, un causal de abandono (Carlino, 2005).

En los casos abordados, los saberes y aprendizajes no quedaron circunscriptos a los aspectos académicos de cada carrera, sino que la experiencia universitaria permitió incorporar nuevos hábitos y conocimientos a la vida cotidiana. En el lapso del primer año aprendieron nuevas palabras, formas de hablar y saberes. En el caso de las estudiantes de Enfermería incorporaron nuevos hábitos y prácticas vinculados al cuidado de la salud. En este sentido, consideramos que es limitante entender los aprendizajes solamente a partir de los resultados obtenidos, por ejemplo, en un examen o según los recorridos alcanzados en la carrera. El trabajo de campo nos permite pensar el saber universitario como un saber mucho más integral que excede los lineamientos curriculares de una materia.

Como pudimos reflejar hasta aquí, en ambas carreras las experiencias y relatos comparten muchas similitudes. Al menos en el ingreso, las dificultades son coincidentes para este grupo de estudiantes como así también el vínculo con el saber, los textos y docentes.

Por último, y a los fines de ahondar en los temas tratados, es indispensable analizar las trayectorias universitarias para profundizar la investigación aquí presentada, como así también desarrollar trabajos empíricos que permitan conocer, comparar y mejorar políticas institucionales preocupadas por la enseñanza y el aprendizaje de quienes ingresan por primera vez al mundo universitario. También consideramos fundamental profundizar las experiencias y prácticas docentes en relación con este tema.

\section{Referencias bibliográficas}

Abramowsky, A. (2014). Maneras de querer: los afectos docentes en las relaciones pedagógicas. $1^{\text {a }}$ ed. - Buenos Aires: Paidós.

Aréchaga, A. J.; et. al. (2015). Puentes hacia una inclusión universitaria: Un análisis a partir de la experiencia en el Curso de Ingreso a las carreras de Sociología de la FaHCE-UNLP (2012-2015). Cuestiones de Sociología (13).

Bourdieu, P. (1997). Capital cultural, escuela y espacio social. México: Siglo XXI.

Bracchi, C. (2016). Descifrando el oficio de ser estudiantes universitarios: entre la desigualdad, la fragmentación y las trayectorias educativas diversificadas. Trayectorias Universitarias, 2 (3).

Carli, S. (2012). El estudiante universitario: Hacia una historia del presente de la educación pública. Buenos Aires, Siglo XXI Editores.

Carlino, P. (2005). Escribir, leer y aprender en la universidad: una introducción a la alfabetización académica. $1^{\text {a }}$ ed. $7^{\text {a }}$ reimp. - Buenos Aires: Fondo de Cultura Económica.

Cerezo, L. (2017). Primeros pasos en la universidad pública de jóvenes en situación de vulnerabilidad social: sus experiencias vinculadas al tiempo, espacio y saberes. Propuesta Educativa, (48),74-82

Colabella, L. y Vargas, P. (2014). La Jauretche. Una universidad en la trama del sur del Gran Buenos Aires. En Gluz, N. et. al. Avances y desafíos en políticas públicas educativas: análisis de casos en Argentina, Brasil, Colombia y Paraguay (251-321). $1^{\text {a }}$ ed. Ciudad Autónoma de Buenos Aires: CLACSO.

Cotignola, M., Legarralde, M. y Margueliche, J. C. (2017). Las trayectorias universitarias de estudiante de Sociología de la FaHCE. Un análisis desde los registros administrativos. Cuestiones de Sociología, 17, e045.

Cuestas, P. (2014). Yo quiero mi título. La perspectiva de los alumnos y alumnas del Plan Fines II de La Plata sobre su paso por esta experiencia educativa. VIII Jornadas de Sociología de la UNLP, 3 al 5 de diciembre de 2014, Ensenada, Argentina.

De la Fare, M.; Rovelli, L. y Atairo, D. (2016). Innovaciones en las políticas nacionales de Educación de Jóvenes y Adultos (EJA): el Programa FINES en Argentina y el PROEJA en Brasil. Horizontes, v. 34, número temático, 7-21, dez. 2016.

Dur I., (2017). Relevamiento de recursos humanos de enfermería en la provincia de Buenos Aires. Evaluación de la carrera de enfermería. Revista Argentina Salud Pública. 8 (32), 38-41.

El Día (11/03/2018). La Escuela de Salud de la UNLP supera a 12 facultades en cantidad de inscriptos. La Plata. https://www.eldia.com/nota/2018-3-11-6-11-50-la-escuela-de-salud-de-la-unlp-supera-a-12-facultades-encantidad-de-inscriptos-la-ciudad\#.WqUKB07E9PQ.whatsapp

Escuela Universitaria de Recursos Humanos del Equipo de Salud (2017). Curso Introductorio 2017.

Ezcurra, A. M. (2007). Los estudiantes de nuevo ingreso: democratización y responsabilidad de las instituciones universitarias. Cuadernos de Pedagogía Universitaria, Universidad Nacional de General Sarmiento, 2007.

(2011). Igualdad en educación superior. Un desafío mundial. Los Polvorines, Argentina: IEC-Universidad Nacional de General Sarmiento.

Finnegan, F. y Brunetto, C. (2015). Contexto, gobierno y actores sociales en la producción de la política de Educación de Jóvenes y Adultos: el plan FinEs Secundaria. En: Pereyra, A. et. al. Prácticas pedagógicas y políticas educativas. Investigaciones en el territorio bonaerense (379-400). $1^{\text {a }}$ ed. Gonnet: UNIPE: Editorial Universitaria.

Garriga Olmo, S. y Torres, M. A. (2018). Acerca del Plan FinEs: un estado de la cuestión (2013-2017). En: Carretero, M. et. al. Nuevos desafíos en educación. Una mirada interdisciplinaria (34-44). $1^{a}$ ed. - Ciudad Autónoma de Buenos Aires: Flacos Argentina. ISBN: 978-950-9379-41-1. Disponible en: https://www.flacso.org.ar/publicaciones/nuevos-desafios-en-educacion-una-mirada-interdisciplinaria/

Gorostiaga, J.; Lastra, K., \& Muiños de Britos, S. M. (2017). Políticas institucionales para favorecer el acceso y la permanencia en universidades argentinas: un análisis de cuatro instituciones del conurbano bonaerense. Páginas De Educación, 10(1), 151-173. https://doi.org/10.22235/pe.v10i1.1363 
Linne, J. (2018). El deseo de ser primera generación universitaria. Ingreso y graduación en jóvenes de sectores populares. Revista Latinoamericana de Educación Inclusiva, 2018, 12 (1), 129-147.

Marradi, A.; Archenti, N. y Piovani, J. I. (2012). Metodología de las ciencias sociales, $2^{\mathrm{a}}$ ed., Buenos Aires, Cengage Learning Argentina.

Más Rocha, S. M. (2019). Políticas institucionales para la inclusión en la universidad. En: Mancovsky, V. y Más Rocha, S. M. (ed.) Por una pedagogía de "los inicios". Más allá del ingreso a la vida universitaria (69-98). $1^{\text {a }}$ ed. Ciudad Autónoma de Buenos Aires: Biblos.

Ministerio de Educación, Presidencia de la Nación (2011). Plan de Finalización de los estudios primarios y secundarios para jóvenes y adultos. Presentación de líneas de acción 2008-2011 del Ministerio de Educación.

Pierella, M. P. (2011). El ingreso a la Universidad como experiencia subjetiva y cultural en estudiantes de la Universidad Nacional de Rosario. Revista Argentina de Educación Superior. Año 3, número 3, ISSN 1852-8171 pp. $26-48$.

(2014). La autoridad en la universidad. Vínculos y experiencias entre estudiantes, profesores y saberes. $1^{\circ}$ ed. Ciudad Autónoma de Buenos Aires: Paidós.

- (2016). Los exámenes en el primer año de la universidad. ¿Instancias de formación o mecanismos de selectividad social? Trayectorias Universitarias, 2(2). Recuperado a partir de https://revistas.unlp.edu.ar/TrayectoriasUniversitarias/article/view/2754

Saraví, G. A. (2015). Juventudes fragmentadas: socialización, clase y cultura en la construcción de la desigualdad. México: Flacso México.

Terigi, F. (2007). "Los desafíos que plantean las trayectorias escolares". III Foro Latinoamericano de Educación Jóvenes y docentes. La escuela secundaria en el mundo de hoy. Fundación Santillana. 